Modeling of sows diurnal activity pattern and detection of parturition using acceleration measurements

Final preprint (uncorrected proof) of article published in Computers and Electronics in Agriculture. Please cite as:

Cornou, C. and Lundbye-Christensen, S. 2012 Modeling of sows diurnal activity pattern and detection of parturition using acceleration measurements. Computers and Electronics in Agriculture $80,97-104$.

DOI: 10.1016/j.compag.2011.11.001 


\title{
Modeling of sows diurnal activity pattern and detection of parturition using acceleration measurements
}

\author{
Cécile Cornou ${ }^{\mathrm{a}, *}$, Søren Lundbye-Christensen ${ }^{\mathrm{b}}$ \\ ${ }^{a}$ University of Copenhagen, Faculty of Life Sciences, Department of Large Animal Science, Grønnegårdsvej \\ 2, DK-1870 Frederiksberg C, Denmark. Tel. +4535333364 Fax. +4535333055 \\ ${ }^{b}$ Department of Cardiology, Cardiovascular Research Center, Aalborg Hospital, Aarhus University \\ Hospital, Sdr. Skovvej 15, 9000 Aalborg, Denmark
}

\begin{abstract}
This article suggests and assesses two different monitoring methods for detecting sows parturition using series of 3-dimensions acceleration measurements previously classified into activity types. Two groups of sows are monitored: a first group $(n=9)$ provided with straw $(\mathrm{S})$, and a second group $(\mathrm{n}=10)$ where no straw is provided (NS); two types of activity are taken into account: High Active behaviour (corresponding to feeding, rooting and nest building behaviours) and Total Active behaviour (including any active activity type). The first method suggests modeling sows' diurnal pattern of activity using a saw-tooth function for the probability of being active and monitoring the series using a Dynamic Generalized Linear Model (DGLM). The second method is based on a cumulative sum of hourly differences of activity, from day-to-day. Both methods use a threshold value, optimized for each group, to detect the onset of farrowing. Best results in terms of sensitivity and specificity are observed for the cumulative sum method, using individual variance and monitoring High Active (sensitivity = $100 \%$; specificity $=100 \%$ ) and Total Active behaviours (sensitivity $=100 \%$; specificity $=95 \%$ ). Results of the DGLM method indicate a sensitivity of $100 \%$ and a specificity of $89 \%$ in average for both group S and NS. Observing the occurrence of alarm times, the DGLM method allows i) earlier detection of farrowing: 15 hours before the onset of farrowing, for both groups, as compared to 9-12 for the other methods; and ii) a better distribution of alarms, i.e. minimize the number of alarms occurring within the last six hours before farrowing.
\end{abstract}

Keywords: Acceleration measurements, Body activity, Dynamic Linear Models, Parturition

\section{Introduction}

In the farrowing house, human attendance during farrowing can help reduce piglets' mortality and improve their weight gain (White et al., 1996). Developing a system that

\footnotetext{
${ }^{*}$ Corresponding author

Email addresses: cec@life.ku.dk (Cécile Cornou ), solc@rn.dk (Søren Lundbye-Christensen)
} 
automatically detects the onset of farrowing would help direct stockman's attention to a specific sow at critical times, without unnecessary work load and disturbances in the farrowing house.

Periparturient activities, especially nest building, and physiological changes are the result of periparturient changes of plasma concentration of hormones, such as prolactin, progesterone and PGF2 $\alpha$ (Algers and Uvnäs-Moberg, 2007; Castren et al., 1993; Rushen et al., 2001). For loose housed and outdoor sows, nest building occurs during the last 24 hours before the onset of parturition, and is most intensive during 12 to $6 \mathrm{~h}$ before farrowing (Algers and Uvnäs-Moberg, 2007). Even though crate-confined sows cannot build nest, they are seen to be highly active the day prior to parturition, performing activities considered as 're-directed' nest building behaviour (or nest building behaviour expressed inappropriately), such as pawing, rooting or mouthing the crate fixtures (Rushen et al., 2001; Hartsock and Barczewski, 1997; Harris and Gonyou, 1998).

The systems that have been developed for detecting the onset of parturition are based on monitoring this re-directed nest building behaviour (or, more generally, an increase in activity) and changes of body temperature. For crate-confined sows $(n=7)$, Bressers et al. (1994) used an radiotelemetric device implanted under the skin close to the ear base, and reported a rise in sows' temperature starting between 6-12 hours before farrowing. Erez and Hartsock (1990) studied changes in one sow's body postures prior to parturition using an infrared photocell system mounted on farrowing crates. They reported an increase in frequency of posture changes beginning 12-24 hours prior to the birth of the first piglet, where a period of intense activity began 6-10 hours before the birth of the first piglet. Oliviero et al. (2008) used two kinds of movement sensors to detect the onset of farrowing: 1) a force sensor was installed under the sows and measured the overall movement of the sows; 2) a photocell was placed at a height of $0.6 \mathrm{~m}$ and detected whether the sow was lying down or standing up. They reported that both sensors produced comparable information about the activity of the animal: the mean time sows spent standing was significantly higher in the 24 hours interval prior to farrowing than in the other 24 hours intervals monitored ( $\mathrm{p}<0.05$, for 10 sows). The force sensor recorded a significant higher number of peaks in the 24 hours interval prior to farrowing than in all the other 24 hours intervals monitored $(\mathrm{p}<0.05$, for 10 sows). Mainau et al. (2009) reported results of Standing Lying Sensors (SLS), consisting of photoelectric cell placed at a height of $72 \mathrm{~cm}$, used to detect position changes for sows prior to farrowing. The SLS was validated using 135 animal-days, and showed a sensitivity of $64 \%$ and a specificity of $88 \%$ in classifying sows posture. They reported a significant increase in postural changes one day before, and on the day of farrowing, with a peak at 0-6 hours prior to farrowing.

Accelerometers have previously been used to monitor the types of activity performed by gestating sows housed in large groups and by sows housed in farrowing crates (Cornou and Lundbye-Christensen, 2008, 2010; Cornou et al., 2011). Results from activity classification in the farrowing house (19 individuals) indicated, among other things, that sows' active behaviours increased significantly $(\mathrm{p}<0.001)$ from 20 to 16 hours before farrowing, and that the number of changes of activity types, indicator of restlessness, increased during the last 24 hours prior to parturition.

This article suggests methods that automatically detect the onset of farrowing. The 
methods use acceleration data that have been previously classified into activity types, as reported in Cornou et al. (2011). After introducing the data, the methods used to model the diurnal pattern and to detect parturition are presented in Section 3. Section 4 presents the results of farrowing detection and Section 5 discusses the methods and results.

\section{Data}

The data set used in this article consists of series of activity types generated from acceleration data, using the automatic classification method suggested by Cornou et al. (2011).

\subsection{Animals, housing and acceleration measurements}

Periparturient sows $(n=24)$ were monitored in the farrowing house of a production herd, in Zealand, Denmark, from May the 27th until June the 13th, 2008. Sows were dry-fed three times daily $(7.15 \mathrm{am}, 12.00 \mathrm{pm}$ and $15.30 \mathrm{pm})$ and kept in crates of dimensions $60 \mathrm{~cm}$ wide and $195 \mathrm{~cm}$ long, inside of a pen of dimension $155 \mathrm{~cm}$ wide and $225 \mathrm{~cm}$ long.

Sows' activity was measured using a 3-dimensions digital accelerometer (LIS3L02DS from STMicroelectronics), at four samples per second, at a range 0-2 g. The exact time of farrowing was registered by use of video recordings. Sows were monitored from their entrance into the farrowing house, and during 7 to 11 days. Half the individuals received approximately $0.5 \mathrm{~kg}$ straw as bedding material every second day (Group S). The rest received no bedding material (Group NS). Acceleration data from 19 sows were available, including 9 sows from group $\mathrm{S}$ and 10 sows from group NS.

\subsection{Qualitative series of activity types}

The quantitative series of acceleration measurements were transformed into qualitative series of activity types using a Multi Process Kalman Filter. For each two minutes period (considered appropriate to recognize one activity and differentiate between activity types; see also Cornou and Lundbye-Christensen (2008)), the classification method provided output as whether the given sow was:

1. HA: High active, corresponding to feeding, rooting, and nest building activities,

2. MA: Medium active, corresponding to standing, sitting or lying sternally, where the sow is active,

3. L1: Lying laterally on one side and passive (sleeping or resting),

4. L2: Lying laterally on the other side and passive (sleeping or resting),

5. LS: Lying sternally and passive (sleeping or resting),

6. LU: Lying in an unclear position, and passive (sleeping or resting); less than $5 \%$ in average for the 19 sows.

Activity types were also grouped into total active $(\mathrm{TA}=\mathrm{HA}+\mathrm{MA})$ and passive $(\mathrm{P}=\mathrm{L} 1+\mathrm{L} 2+\mathrm{LS}+\mathrm{LU})$ categories. Results from the classification method indicated a sensitivity ranging from 75 to $100 \%$ when classifying activity types, and from 96 to $100 \%$ when collapsing into active vs. passive categories.

A detailed description of the experimental protocol and the classification method is found in Cornou et al. (2011). 


\section{Modeling and monitoring activity}

Two methods are suggested to model and monitor sows activity. Both methods are based on two minutes periods of dichotome data (active/passive) for a specific sow.

- In the first model a dynamic generalized linear binomial model (DGLM, see West and Harrison (1997)) is applied for describing the smoothly changing probability for being active in each two minute period. The diurnal pattern is incorporated herein as a parametric model in which parameters vary between sows. Application of the non-linear Kalman filter yields summary variables indicating changes in pattern.

- The second method is based on hourly sums of two minutes periods of active and passive separately. The diurnal pattern is dealt with by subtracting the present hourly sum from the corresponding hourly sum on the previous day. Thereafter, the cumulative sum (CUSUM) of these differences is calculated.

Both methods take into account the diurnal pattern, either by modeling it (first method) or by monitoring the hourly activity from one day as compared to the same hour of the previous day (second method).

\subsection{Modeling of diurnal pattern}

The average diurnal pattern is quantified using the period preceding farrowing, i.e. representing a normal, or reference behaviour. Assuming that behavioural changes occur up to 24 hours before the onset of farrowing, the reference days for modeling diurnal pattern are from the day of entrance to the farrowing house until $d_{-2}$ (with $d_{0}$ being the farrowing day). This period represents a total of 70 days, for the 19 sows.

Explorative plots of two minutes periods of total active behaviours, value=1, vs. total passive, value $=0$, showed a regular diurnal pattern of active behaviours, corresponding to the times where sows were fed. The diurnal pattern varies between sows, but many features such as peaks of activity, located around the feeding times are shared between sows. This indicates a parametric model with parameters varying between sows.

The binary series for group S and NS were smoothed (using smooth. spline from the software R). The results of smoothing are shown with solid lines, group S (black solid line) and NS (grey solid line), in Figure 1. Hereby three dominant peaks located around feeding times and a less marked active period around evening were revealed. A notably variation between sows was also seen in the activity pattern of evening (feeding time and later).

The average activity level differed between the two groups, as did the time of peaks. A higher level of activity for group S (as compared to group NS) is observed early in the day (from 6 am to $1 \mathrm{pm}$ ), while sows from group NS appear slightly more active later in the day (from $5 \mathrm{pm}$ to $10 \mathrm{pm}$ ). This is in accordance with the findings by Cornou et al. (2011).

The visual inspection of time series for each sow showed further that the general activity level and also the high/low activity contrast vary between the sows. This inhomogeneity is addressed in the modeling of the diurnal pattern. 
The four peaks of activity are modeled, for the respective two groups, by the sawtooth function, increasing linearly from zero to one and decreasing to zero, where timepoints of start, peak, and end are given.

A generalized linear model for the binomial family was fitted for each group, using the glm function of the software R. Let $\pi_{t}$ be the probability of an "average" sow being active at time $t$, then

$$
\operatorname{logit} \pi_{t}=\log \pi_{t} /\left(1-\pi_{t}\right)=\alpha_{0}+\alpha_{1} X_{1}(t)+\alpha_{2} X_{2}(t)+\alpha_{3} X_{3}(t)+\alpha_{4} X_{4}(t) .
$$

The average diurnal patterns for group S and NS obtained from (1) are plotted with dotted lines in Figure 1.

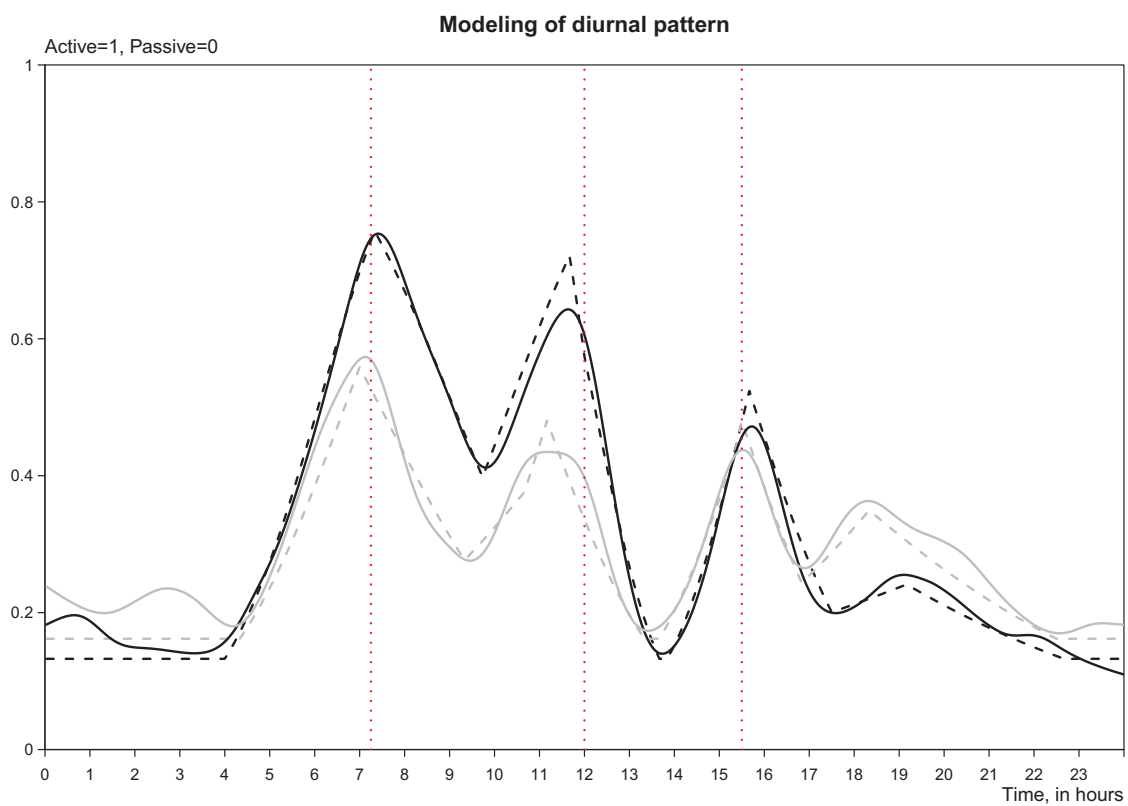

Figure 1: Modeling of the diurnal pattern of active behaviours (TA). Solid lines: smoothed lines of 2 min classified observations for the group S (black, 9 sows) and NS (grey, 10 sows). Dotted lines: result of the model describing the diurnal pattern (1), $D_{S}$ for the group $\mathrm{S}$ (black) and $D_{N S}$ for the group NS (grey). The vertical dotted lines indicate feeding times $(7.15 \mathrm{am}, 12.00 \mathrm{pm}$ and $15.30 \mathrm{pm})$.

By letting the daily pattern $D(t)=\alpha_{0}+\alpha_{1} X_{1}(t)+\alpha_{2} X_{2}(t)$ and the evening pattern $E(t)=\alpha_{3} X_{3}(t)+\alpha_{4} X_{4}(t)$, where $\alpha_{0}, \ldots, \alpha_{4}$ are obtained from fitting (1) to group $\mathrm{S}$ and NS, respectively, we obtain the average diurnal pattern for each group as $D(t)+E(t)$.

The previously described variation between sows in diurnal pattern within the same group is obtained by letting

$$
\operatorname{logit} \pi_{j t}=\beta_{0 j}+\beta_{1 j} D(t)+\beta_{2 j} E(t),
$$

where $\pi_{j t}$ is the probability of the $j$ th sow being active at time $t, \beta_{0 j}$ is the individual 
activity level, and $\beta_{1 j}, \beta_{2 j}$, describes the high/low activity contrast characterizing the $j$ th sow. The average behaviour is described by letting $\beta_{0 j}=0$ and $\beta_{1 j}=\beta_{2 j}=1$.

Table 1 indicates the time of the day where the four activity peaks occur for the group S (left panel) and NS (right panel). Mean values, standard deviations, and range (minimum and maximum values) for the coefficients $\alpha$ 's from (1) and $\beta$ 's from (2) are also reported.

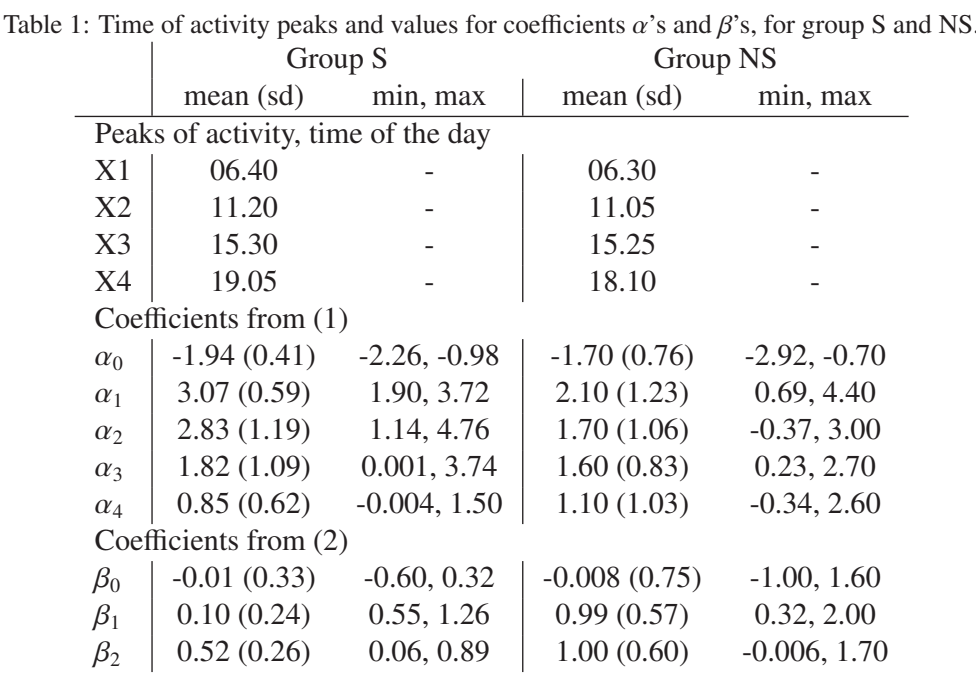

The mean standard deviation for the coefficients $\alpha$ 's and $\beta$ 's as well as the larger range of values (min, max) tend to indicate a larger inhomogeneity of diurnal pattern for sows within the group NS.

\subsection{First method: Logistic dynamic generalized linear model for diurnal variation}

The discrete-valued times series of the active category, $\mathrm{TA}=1$ vs. $\mathrm{TA}=0,\left\{y_{t}\right\}$, which for the given probability $\pi_{t}$ follows a Bernoulli distribution can be described as

$$
p\left(y_{t} \mid \pi_{t}\right)=\pi_{t}^{y_{t}}\left(1-\pi_{t}\right)^{1-y_{t}}, \quad y_{t}=0,1,2, \ldots, n_{t} ; \quad n_{t}=0,1 ; \quad 0<\pi_{t}<1 .
$$

The logit transformation, $\eta_{t}=\log \pi_{t} /\left(1-\pi_{t}\right)$, maps $\pi_{t}$ to the linear predictor $\eta_{t}$ (Hosmer and Lemeshow, 2000; Triantafyllopoulos, 2009).

A dynamic generalized linear model (DGLM) for binomial data (West and Harrison, 1997) is implemented on the times series $\left\{y_{t}\right\}$. The observation equation (4) describes the impact of the underlying latent process $\theta_{t}$ on the linear predictor $\eta_{t}$.

$$
\begin{aligned}
\eta_{t} & =X_{t}^{\top} \theta_{t}, \\
\theta_{t} & =\theta_{t-1}+w_{t} .
\end{aligned}
$$

The design matrix $X_{i t}^{\top}=\left(1, D_{i t}, E_{i t}\right)$ describes the diurnal pattern of the series for the group $i=\{N, N S\}$ in accordance with (2). The individual activity level and high/low 
activity contrast are contained in $\theta_{t}$ which is allowed to vary between sows and also over time.

The evolution over time of $\theta_{t}$ is described in the system equation (5), where the error terms $w_{t}$ are assumed normally distributed with mean 0 and variance-covariance matrix $W$, assumed known and constant. Hereby the process $\theta_{t}$ is a three dimensional random walk. The size of the entries of $W$ reflects the degree of smoothness of the trajectories of $\theta_{t}$. After visual observation of preliminary results, the evolution matrix $W$ is set to be diagonal with diagonal entries $10^{-4}$.

The initial values describing the distribution between sows are set as

$m_{0}=\left(\begin{array}{l}0 \\ 1 \\ 1\end{array}\right), \quad C_{0, S}=\left(\begin{array}{ccc}0.11 & 0.04 & 8.6^{-5} \\ 0.04 & 0.06 & 0.08 \\ 8.6^{-5} & 0.08 & 0.24\end{array}\right), \quad C_{0, N S}=\left(\begin{array}{ccc}0.57 & 0.30 & 0.11 \\ 0.30 & 0.33 & 0.25 \\ 0.11 & 0.25 & 0.36\end{array}\right)$

with identical value for $m_{0}$ for the two groups, and values for the sampling variance matrices $C_{0, S}$ and $C_{0, N S}$ obtained from the sample variation of the fitted values of $\beta_{i}$ $(i=\{N, N S\})$ from fitting (2) to data.

\subsubsection{Estimating the individual pattern}

For each new observation $y_{t}$ the updating equations of the DGLM estimates the mean $m_{t}$ and the sampling variance matrix $C_{t}$ of the underlying value $\theta_{t}$. This will be denoted $\left(\theta_{t} \mid D_{t}\right) \sim\left[m_{t} ; C_{t}\right]$, where $D_{t}=\left\{y_{1}, \ldots, y_{t}\right\}$.

Letting $\left(\theta_{t-1} \mid D_{t-1}\right) \sim\left[m_{t-1} ; C_{t-1}\right]$ be given by recursion starting with $\left(\theta_{0} \mid D_{0}\right) \sim$ $\left[m_{0} ; C_{0}\right]$, see (6), the prior distribution of $\theta_{t}$ is obtained from (5).

$$
\left(\theta_{t} \mid D_{t-1}\right) \sim\left[a_{t} ; R_{t}\right], \quad a_{t}=m_{t-1}, R_{t}=C_{t-1}+W .
$$

Hence, from (4) the prior for the linear predictor is

$$
\left(\eta_{t} \mid D_{t-1}\right) \sim\left[f_{t} ; q_{t}\right], \quad f_{t}=X_{t}^{\top} a_{t}, q_{t}=X_{t}^{\top} R_{t} X_{t} .
$$

These moments correspond to the following beta-distribution for the probability of being active at time $t$;

$$
\left(\pi_{t} \mid D_{t-1}\right) \sim \operatorname{Beta}\left(r_{t}, s_{t}\right), \quad r_{t}=\frac{1+\exp \left(f_{t}\right)}{q_{t}}, \quad s_{t}=\frac{1+\exp \left(-f_{t}\right)}{q_{t}} .
$$

Updating for $y_{t}$ yields

$$
\left(\pi_{t} \mid D_{t-1}\right) \sim \operatorname{Beta}\left(r_{t}^{*}, s_{t}^{*}\right), \quad r_{t}^{*}=r_{t}+y_{t}, \quad s_{t}^{*}=s_{t}+1-y_{t} .
$$

The updated distribution of the linear predictor is then approximated by

$$
\left(\eta_{t} \mid D_{t}\right) \sim\left[f_{t}^{*} ; q_{t}^{*}\right], \quad f_{t}^{*}=\log \frac{r_{t}^{*}}{s_{t}^{*}}, \quad q_{t}^{*}=\frac{1}{r_{t}^{*}}+\frac{1}{s_{t}^{*}} .
$$

The posterior for the latent process is

$$
\left(\theta_{t} \mid D_{t}\right) \sim\left[m_{t} ; C_{t}\right], \quad m_{t}=a_{t}+\frac{R_{t} X_{t}}{q_{t}}\left(F_{t}-f_{t}\right), C_{t}=R_{t}-\frac{R_{t} X_{t} X_{t}^{\top} R_{t}}{q_{t}^{2}}\left(q_{t}-Q_{t}\right) .
$$


Hence, at a given time $t$, approximations of the general level, the day and the extra evening contrasts, characterizing the individual sow conditional on data up to $y_{t}$ are given by the elements of $m_{t}$. For further details concerning these calculations we refer to West and Harrison (1997).

Figure 2 illustrates the implementation of the DGLM on the series of the active category for sow 1 , using the diurnal pattern for the group $S, D_{S}$. The solid black line is a smoothed representation of the binomial series of TA category (using smooth.spline from the software R); the higher level of activity prior farrowing (at day 1, on the horizontal axis) is clear.

The dotted line represents the underlying mean of the observed series, using a DGLM with evolution variance $W=10^{-4}$, i.e. a dynamic model. The higher activity level observed before farrowing is well followed by an increase of the underlying mean; this is especially observed few hours before the onset of farrowing (represented by the vertical dotted line).

Finally, the solid grey line represents the underlying mean of a static model, i.e. using evolution variance $W=0$; here the underlying mean shows a very stable pattern through the entire period, even when the observed activity level increases.

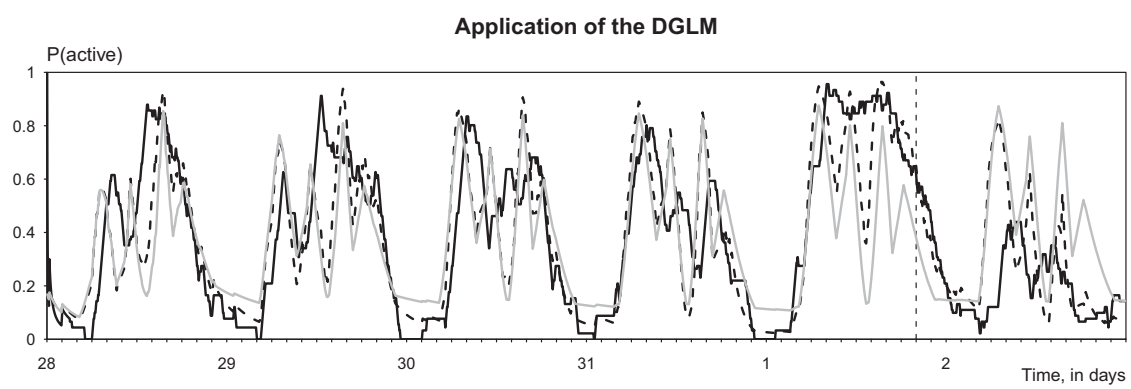

Figure 2: Implementation of the DGLM on the series for sow 1 of the group S (May the 28th to June the 2nd). Solid black line: smoothed series of active behaviour; dotted black line: underlying mean using evolution variance $W=10^{-4}$; solid grey line: underlying mean using evolution variance $W=0$. The vertical dotted line indicates the onset of farrowing.

\subsubsection{Monitoring the DGLM}

The time series plots of the components of the estimated latent process, $m_{t}$, clearly show an increasing pattern in the hours preceding farrowing. However, since the level of each component is characteristic to the individual sow, changes relative to the (unknown) sow specific values rather than the values themselves are of interest. Hence a global cut-off level to indicate an alarm is not feasible. Instead we suggest another approach in which we fit two models concomitantly:

- a dynamic model (MD) with evolution variance $W=10^{-4}$,

- a static model (MS) with $W=0$. 
The static model follows the dynamic model as long as the diurnal pattern stays relatively stable between days. When the sow changes pattern, the static DGLM fails to follow and fit the data whereas the dynamic model is capable of adapting $m_{t}$ according to the change in pattern. Therefore the static model will serve as the reference from which deviations are monitored before onset of farrowing.

To formalize, we calculate the normalized distance as

$$
D S_{t}=\Delta_{t}^{\top} C_{t}^{-1} \Delta_{t}, \quad \Delta_{t}=m_{t}^{M D}-m_{t}^{M S},
$$

where $m_{t}^{M D}$ and $m_{t}^{M S}$ are obtained from fitting the dynamic and the static DGLM, and $C_{t}$ is obtained from the static model, according to (12).

The differences $\Delta_{i t}$ are calculated for each sow of the corresponding group $i=$ $\{N, N S\}$ and thereafter monitored using a threshold value $T D_{i}$.

Optimization of the threshold is performed for each group in the range [500,1000] by steps of 10; threshold optimization range and step were chosen after a first visual inspection of the resulting plots. An alarm is given each time $D S_{i t}$ crosses over the threshold $T D_{i}$.

\subsection{Second method: Modeling of activity using a cumulative sum based on daily vari- ation}

The second method suggested is based on monitoring the cumulative sum (CUSUM) of differences of total active behaviours at a given hour, from day to day.

Each hourly sum of 2 min series at time $t, S_{t}$, is subtracted to the corresponding hour on the previous day, $S_{t-24}$. The differences, $\delta_{t}=S_{t}-S_{t-24}$, are thereafter standardized by dividing them by their standard deviation. Assuming a negligible correlation between $S_{t}$ and $S_{t-24}$, the standard deviation of $\delta$ is approximated using $\sqrt{2 V_{i}}$. The variance $V_{i}$ is calculated for each group, with $i=\{\mathrm{N}, \mathrm{NS}\}$, from 24 hours after the entrance to the farrowing house, and up to 48 hours before the onset of farrowing; individual variance, $V_{j}$, is also calculated for each sow, with $\mathrm{j}=\{1, \ldots, 19\}$, for the same time interval.

The cumulative sum, $C S$, of the standardized series is then calculated with each group respective variance $V_{i}$ as

$$
C S_{i t}=\sum_{25}^{t} \delta_{i t} / \sqrt{2 V_{i}} .
$$

In order to take into account potential variations in amplitude between sows, cumulative sum for individually standardized series $C S_{j t}$ is also calculated. Individual variance $V_{j}$ are calculated using series corresponding to each sow (instead of all series corresponding to the respective groups).

Figure 3 illustrates, for sow 1, the daily differences for every hour $t$, of the sums of 2 min total active behaviours (in bars), and the cumulative sum of these differences (plain lines). The cumulative sums are standardized with the variance of group S. 


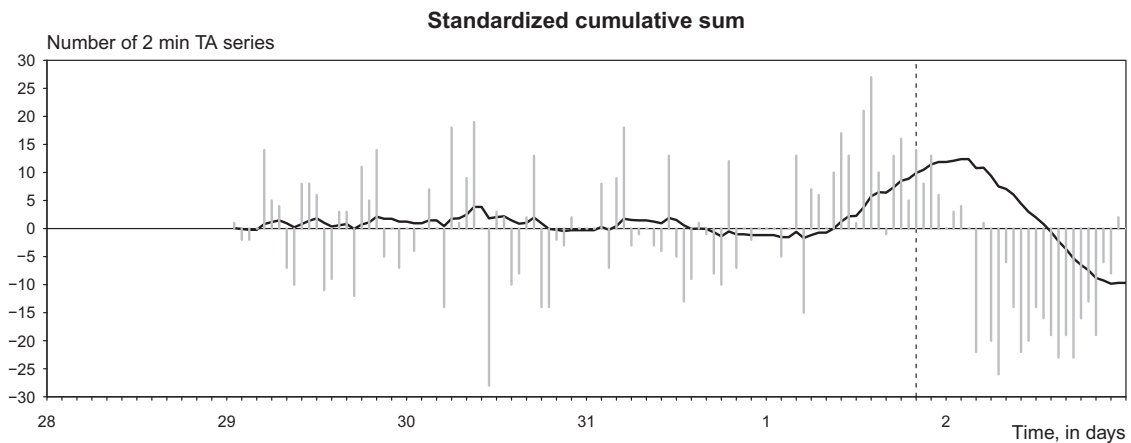

Figure 3: In bars: Hourly differences for TA behaviours for sow 1 of the group S (May the 28th to June the 2nd). The plain lines show the cumulative sums of the daily differences; the cumulative sums are standardized using the variance for group $\mathrm{S}$. The vertical dotted lines indicate the onset of farrowing.

An increase of hourly differences of activity, as indicated by the grey vertical bars, is observed from about 10 to 12 hours before the onset of farrowing. This activity increase is well revealed by the standardized cumulative sum, which shows a positive trend beginning 10-12 hours before the onset of farrowing.

\subsubsection{Monitoring the CUSUM}

The increase of $C S_{i t}$ is monitored using a threshold value $T C_{i}$ optimized for each group $i=\{N, N S\}$. The increase of the cumulative sums of individually standardized series, $C S_{j t}$, is monitored using a single threshold value $T C$.

An alarm is given each time $C S_{i t}$ crosses over $T C_{i}$ and $C S_{j t}$ crosses over $T C$. Optimization is performed in the range $[3,12]$ by steps of 1 ; optimization range and step were chosen after a first visual inspection of the resulting plots.

\subsection{Evaluation of the results}

A True Positive, TP, is when at least one alarm occurs within the last 24 hours preceding the onset of farrowing. A False Positive, FP, is when at least one alarm is given before the last 24 hours preceding the onset of farrowing. A False Negative, $\mathrm{FN}$, is when no alarm is given within the last 24 hours preceding the onset of farrowing, while a True Negative, TN, is when no alarm is given before the last 24 hours preceding the onset of farrowing.

Optimization is based on maximizing the sum of the sensitivity, $\mathrm{TP} /(\mathrm{TP}+\mathrm{FN})$, and the specificity, $\mathrm{TN} /(\mathrm{TN}+\mathrm{FP})$.

Performance of the detection methods are thereafter assessed using sensitivity, specificity and error rate, $\mathrm{FP} /(\mathrm{FP}+\mathrm{TP})$. 


\section{Results}

\subsection{DGLM}

Figure 4 illustrates the application of the method for monitoring parturition using the DGLM, for sow 1. The solid line represents the time plot of $D S_{t}$, as defined in (13). Despite the fact that the distances $D S_{t}$ are standardized, graphical outputs indicate that the amplitude of fluctuations in $D S_{t}$ within the last 24 hours before farrowing varies between sows.

The increase of activity is here monitored using the optimized threshold value for group $\mathrm{S}, T D_{S}=870$. The vertical line indicates the onset of farrowing for sow 1 .

Monitoring activity deviation using the DGLM

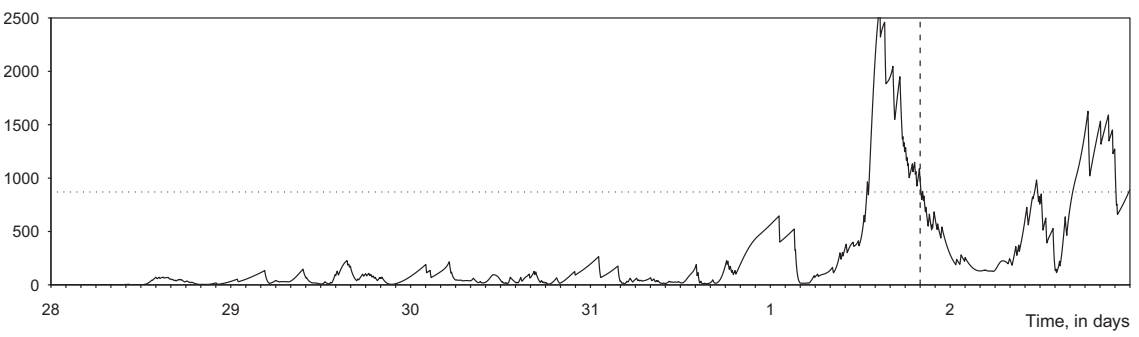

Figure 4: Monitoring activity deviation using the DGLM for sow 1 of the group S (May the 28th to June the 2 nd). The solid line shows the difference of underlying mean between the dynamic $\left(W=10^{-4}\right)$ and static models $(W=0)$; the horizontal dotted line indicates the threshold value of 870 , used for farrowing detection for the group $\mathrm{S}$. The vertical dotted line indicates the onset of farrowing.

Table 2 shows the results of the detection method using the DGLM, and the optimized threshold values of $T D_{S}=870$ for group $S$ and $T D_{N S}=520$ for group NS, and the total results for both groups. The onset of farrowing is detected for all 19 sows, resulting in 100\% sensitivity; false alarms are observed for 2 sows (one of each group, at 42 and 80 hours before the onset of farrowing), resulting in $89 \%$ specificity. Farrowing is detected in average 15 hours before the onset of farrowing, for both groups. The majority of alarms occur between 12 to 24 hours before the onset of farrowing.

\subsection{CUSUM}

Table 3 shows the results for the cumulative sum (CUSUM) using TA behaviours and group variance (left panel) and individual variances (right panel), using the optimized threshold values for each group.

Using group variances, results indicate that the onset of farrowing is detected for 8 out of 9 sows of group S, and for all 10 sows of group NS, in average 9.9 and 13.1 hours before farrowing, respectively. Two sows of the group NS are classified as FP. One of these sows releases two alarms, at 77 and 30 hours before farrowing; the other sow releases an alarm at 85 hours before farrowing. For the sow not detected, an alarm is observed 1 hour after the onset of farrowing. The time of farrowing detection ranges from 0 to 22 hours before farrowing. 
Table 2: Results of farrowing detection using the DGLM method based on TA behaviours, using a threshold for group S and for group NS and in total. The lower part shows the mean time for the alarms ( $\pm \mathrm{sd})$, and distribution of numbers of alarms into four periods before the onset of farrowing.

\begin{tabular}{l|ccc} 
& $\mathrm{S}(\mathrm{n}=9)$ & $\mathrm{NS}(\mathrm{n}=10)$ & Total Group \\
\hline Threshold TD & 870 & 520 & - \\
\hline True Positive & 9 & 10 & 19 \\
True Negative & 8 & 9 & 18 \\
False Positive & 1 & 1 & 2 \\
False Negative & 0 & 0 & 0 \\
Sensitivity & 100 & 100 & 100 \\
Specificity & 89 & 90 & 89 \\
Error rate & 10 & 9 & 10 \\
\hline Mean ( \pm SD), in hours & $15.0(4.3)$ & $15.0(7.5)$ & $15.0(6.0)$ \\
\hline Alarm [0-6[ hours & 0 & 1 & 1 \\
Alarm [6-12[ hours & 2 & 2 & 4 \\
Alarm [12-18[ hours & 5 & 3 & 8 \\
Alarm [18-24[ hours & 2 & 4 & 6
\end{tabular}

Table 3: Results of farrowing detection for the cumulative sum method based on TA behaviours using sows' group variances (left panel) and individual variances (right panel), for group S, group NS and in total. The lower part shows the mean time for the alarms $( \pm \mathrm{sd})$, and distribution of numbers of alarms into four periods before the onset of farrowing.

\begin{tabular}{l|ccc|ccc} 
& \multicolumn{3}{|c}{ Group variances } & \multicolumn{3}{c}{ Individual variances } \\
& $\mathrm{S}(\mathrm{n}=9)$ & $\mathrm{NS}(\mathrm{n}=10)$ & Total & $\mathrm{S}(\mathrm{n}=9)$ & $\mathrm{NS}(\mathrm{n}=10)$ & Total \\
\hline Threshold TC & 9 & 6 & - & 9 & 6 & - \\
\hline True Positive & 8 & 10 & 18 & 9 & 10 & 19 \\
True Negative & 9 & 8 & 17 & 9 & 9 & 18 \\
False Positive & 0 & 2 & 2 & 0 & 1 & 1 \\
False Negative & 1 & 0 & 1 & 0 & 0 & 0 \\
Sensitivity & 89 & 100 & 95 & 100 & 100 & 100 \\
Specificity & 100 & 80 & 89 & 100 & 90 & 95 \\
Error rate & 0 & 17 & 10 & 0 & 9 & 5 \\
\hline Mean ( \pm SD), in hours & $9.9(5.0)$ & $13.1(9.1)$ & $11.7(7.5)$ & $8.7(5.8)$ & $14.8(4.9)$ & $11.9(6.0)$ \\
\hline Alarm [0-6[ hours & 1 & 4 & 5 & 3 & 0 & 3 \\
Alarm [6-12[ hours & 5 & 0 & 5 & 3 & 4 & 7 \\
Alarm [12-18[ hours & 2 & 1 & 3 & 3 & 0 & 3 \\
Alarm [18-24[ hours & 0 & 5 & 5 & 0 & 6 & 6
\end{tabular}


When standardizing each series with sow's individual variance using (14), all 19 sows are detected, in average 8.7 (group S) and 14.8 (group NS) hours before farrowing. Only one FP is observed for group NS, at 84 hours before farrowing; the same sow for which alarm was observed 85 hours before farrowing using group variance and 80 hours using the DGLM method. The time of farrowing detection ranges from 0 to 19 hours before farrowing.

The cumulative sum method was also applied for the high active (HA) activity type, using both group variances and individual variances and following the same procedure as for TA (data not shown).

Applying group variances, results indicate that the onset of farrowing is detected for all 9 sows of group $\mathrm{S}$ and for 8 out of 10 sows from group NS, in average 9.1 and 12.6 hours before farrowing, respectively. One sow of group S is classified as FP. This sow releases two false alarms, at 106 and 46 hours before farrowing. For the two sows not detected (FN), alarms are given 1 and 5 hours after the onset of farrowing. The distribution of the alarm times indicates that most of the sows are detected in the interval [6-12 [ hours before farrowing $(n=10)$. The time of farrowing detection ranges from 5 to 21 hours before farrowing.

Using individual variances, results indicate both a sensitivity and specificity of $100 \%$. Sows are detected in average 7.4 and 10.9 hours before farrowing. The alarm times are homogenously distributed within the four 6 hours intervals before farrowing. The time of farrowing detection ranges from 0 to 18 hours before farrowing.

\section{Discussion}

Two methods for detecting the onset of farrowing are suggested. Both methods take into account the diurnal pattern of active behaviours observed around feeding time. In the first method, the diurnal pattern is modeled and included in the latent process of a generalized dynamic linear model (DGLM). In the second method, the diurnal pattern is taken into account by subtracting the activity observed at a given hour, to the same hour of the previous day. For both methods, alarms are given when individual sow's activity (either TA or HA) crosses over a given threshold, optimized both for the group provided with straw (S) and the group receiving no straw (NS).

The best results, in terms of sensitivity and specificity are observed using the method based on cumulative sum of hourly differences for HA activity type, and using sows' individual variance: all sows are detected, and no false alarm occurs. i.e. both sensitivity and specificity are $100 \%$.

The method based on a DGLM results in a sensitivity of $100 \%$ and a specificity of $89 \%$ in average for both groups. As compared to the methods based on the cumulative sum of hourly differences, the average time for farrowing detection based on the DGLM is earlier, i.e. there is more time between alarm and farrowing onset: 15 hours in average for both groups, as compared to 9 to 12 hours for the other methods.

Threshold optimization was here based on maximizing the sum of the sensitivity and specificity. Alternatively, sensitivity and specificity could be given different weights: if the cost of a false alarm (time spent to check a sow, risk of not reacting to false alarms if too many) is considered negligible, the methods' sensitivity could be given more weight in the process of optimizing the threshold. 
Using a same threshold value for both groups was attempted for the DGLM method. A threshold that reduced the number of false positive to 1 (as being the minimum observed when optimizing the threshold per group), resulted in 2 false negatives. The parameters describing the diurnal pattern of these two sows indicated that one of these sows had the highest general activity level, while the other sow had the lowest general activity level.

When different thresholds gave the same sensitivity and specificity values, the smallest threshold was chosen, in order to get the earliest possible alarms. For practical application, it can be argued that earlier detection is to be preferred, in order to give the opportunity to the stockman to make the best use of this information, e.g. preparing the crate or checking on the sow. Hence, there should be as few as possible alarms in the last 6 hours before the onset of farrowing. Observing the distribution of alarm times for the different methods, both the DGLM and the cumulative sum using HA and group variance gave the best distributed alarms: fewer alarms occurred in the intervals [0-6[ and [18-24[ hours and most alarms occurred in [6-18[ hours before farrowing.

The approach chosen to assess the methods' performance was based on two periods: the period preceding the last 24 hours before farrowing, and the last 24 hours before the onset of farrowing. For the CUSUM method that used group variance, two distinct sows (one of group S and one of group NS) released each two false alarms. In the suggested approach, each of this sow was considered as one false positive. As this experiment included a limited number of sows, this was considered a reasonable way of assessing the detection methods. However, it may have slightly increased the performance of the methods and a more correct approach should be chosen while performing a larger scale experiment. An alternative could be to divide the entire period into 24 hours intervals and look for alarms within each interval.

Potential application of the DGLM method requires investigating the possibilities of adjustment for individual herds feeding time. The method for modeling diurnal pattern suggested in this article included four peaks of activity, where three of them were associated with herd feeding times. A further experiment is needed to assess whether adjusting parameters of the saw-tooth function with other feeding times is straight forward.

In order to estimate group and/or individual variance, the methods suggested used some reference days, as being the days from the entrance to the farrowing house and up to 48 hours before the onset of farrowing. These reference days ranged from 1 to 6 : 1 day for 1 sow, 2 days for 2 sows; 3 for $3 ; 4$ for 10; 5 for 2; and 6 days for 1 sow. It can be argued that the use of individual parameters, as used in both the DGLM and the CUSUM method with individual variances, are over optimized because the reference days were known beforehand. Since sows are generally transferred to the farrowing house only few days before the onset of farrowing, the detection methods should adjust as fast as possible to the sows' individual levels. The use of average parameters is therefore to be preferred, and could still differ according to e.g. the quantity of straw provided to the sow.

The access to bedding material seems to influence the starting time and quantity of preparturient activity. This was observed for the CUSUM methods, both using group and individual variances. Sows without access to straw start performing preparturient activity earlier and are therefore detected at an earlier stage. However, the impact of 
access to straw on alarm time was not observed using the DGLM method.

Results of this experiment are in accordance with previous studies which reported increase of activity during the last 24 hours before farrowing (Erez and Hartsock, 1990; Oliviero et al., 2008) and a peak of more intense activity during the last 6 hours preceding the onset of farrowing (Mainau et al., 2009; Oliviero et al., 2008). These studies used infrared photocells, force sensors or Standing Lying Sensors, i.e. techniques adapted only to crates. The advantage of accelerometer measurements is that it can be used for loose-housed farrowing. Acceleration measurement in loose systems should therefore be tested to fully optimize the use of this technique. Data collection using an ear chip instead of a neck collar (as used in this experiment) should also be tested, as it is more realistic for practical use on production farms.

Other types of activities classified using acceleration measurements include for instance lying laterally and lying sternally (Cornou et al., 2011); deviations in these types of activity could also be included in a developing a method for detecting the onset of parturition and/or other behavioural deviations indicating health disorders. Whereas the suggested methods reduced data to binary distribution, the incorporation of different activity types would call for a multinomial distribution.

A larger scale experiment should take into account factors as sow parity and influence of the farrowing time (night or day); the adaptation of the methods to other feeding times (especially for the DGLM method) should also be investigated. Threshold optimization was here based on solely 19 sows. The optimization process should also include a larger number of sows if the method should be applied in practice. It should focus on finding a single threshold, which could potentially differ according to e.g. the housing system.

\section{Conclusion}

In conclusion, the detection methods for farrowing suggested in this article showed sensitivity ranging from 89 to $100 \%$ and specificity ranging from 80 to $100 \%$. Best results in terms of sensitivity and specificity are observed for the CUSUM method, using individual variance and monitoring High Active (sensitivity $=100 \%$; specificity $=100 \%$ ) and Total Active behaviours (sensitivity $=100 \%$; specificity $=95 \%$ ). Results of the DGLM method indicate a sensitivity of $100 \%$ and a specificity of $89 \%$ in average for both group S and NS. Observing the occurrence of alarm times, the DGLM method allows i) earlier detection of farrowing: 15 hours before the onset of farrowing, for both groups, as compared to 9-12 for the other methods; and ii) a better distribution of alarms, i.e. minimize the number of alarms occurring within the last six hours before farrowing. A larger scale experiment should test the use of an ear-chip to monitor sow activity and assess whether factors such as e.g. time of the day when farrowing occurs (day or night) or parity affect the time of detection of the onset of farrowing.

\section{Acknowledgements}

The authors gratefully acknowledge the staff at Askelygård, the Danish Pig Production and the Department of Computer Science of Copenhagen University, for assistance with data collection. 


\section{References}

Algers, B., Uvnäs-Moberg, K., 2007. Maternal behavior in pigs. Horm. Behav. 52, $78-85$.

Bressers, H. P. M., te Brake, J. H. A., Jansen, M. B., Nijenhuis, P. J., Noordhuizen, J. P. T. M., 1994. Monitoring individual sows: radiotelemetrically recorded ear base temperature changes around farrowing. Livest. Prod. Sci. 37, 353-361.

Castren, H., Algers, B., de Passillé, A. M., Rushen, J., Uvnäs-Moberg, K., 1993. Preparturient variation in progesterone, prolactine, oxytocin and somatostatin in relation to nest building in sows. Appl. Anim. Behav. Sci. 38, 91-102.

Cornou, C., Lundbye-Christensen, S., 2008. Classifying sows' activity types from acceleration patterns: an application of the multi-process kalman filter. Appl. Anim. Behav. Sci. (111), 262-273.

Cornou, C., Lundbye-Christensen, S., 2010. Classification of sows' activity types from acceleration patterns using univariate and multivariate models. Comput. Electron. Agric. (72), 53-60.

Cornou, C., Lundbye-Christensen, S., Kristensen, A., 2011. Modeling and monitoring sows' activity types in the farrowing house using acceleration data. Comput. Electron. Agric. (76), 316-324.

Erez, B., Hartsock, T. G., 1990. A microcomputer-photocell system to monitor periparturient activity of sows and transfer data to a remote location. J. Anim. Sci. 68, $88-94$.

Harris, M., Gonyou, H. W., 1998. Increasing available space in a farrowing crate does not facilitate postural changes or maternal responses in gilts. Appl. Anim. Behav. Sci. 59, 285-296.

Hartsock, T. G., Barczewski, R. A., 1997. Prepartum behavior in swine: effects of pen size. J. Anim. Sci. 75, 2899-2904.

Hosmer, D. W., Lemeshow, S., 2000. Applied Logistic Regression, 2nd Edition. Chichester, Wiley, New York.

Mainau, E., Dalmau, A., de-la Torre, J. R., Manteca, X., 2009. Validation of an automatic system to detect position changes in puerperal sows. Appl. Anim. Behav. Sci. $121,96-102$.

Oliviero, C., Pastell, M., Heinonen, M., Heikkonen, J., Valros, A., Ahokas, J., Vainio, O., Peltoniemi, O. A. T., June 2008. Using movement sensors to detect the onset of farrowing. Biosystems Eng. 100 (2), 281-285.

Rushen, J., Robert, S., Farmer, C., 2001. Evidence of a limited role for prolactine in the preparturient activity of confined gilts. Appl. Anim. Behav. Sci. 72, 309-319. 
Triantafyllopoulos, K., 2009. Inference of dynamic generalized linear models: On-line computation and appraisal. Int. Stat. Rev. 77, 430-450.

West, M., Harrison, J., 1997. Bayesian Forecasting and Dynamic Models, 2nd Edition. Springer, New York, USA.

White, K. R., Anderson, D. M., Bate, L. A., December 1996. Increasing piglet survival through an improved farrowing management protocol. Canadian Journal of Animal Science 76 (4), 491-495. 